\title{
Relevance of matrix metalloproteases in non-small cell lung cancer diagnosis
}

\author{
Sonia Blanco-Prieto ${ }^{1}$, Leticia Barcia-Castro', María Páez de la Cadena , Francisco Javier Rodríguez-Berrocal ${ }^{1}$, \\ Lorena Vázquez-Iglesias ${ }^{1}$, María Isabel Botana-Rial ${ }^{2}$, Alberto Fernández-Villar ${ }^{2}$ and Loretta De Chiara ${ }^{1 *}$
}

\begin{abstract}
Background: The need for novel biomarkers that could aid in non-small cell lung cancer (NSCLC) detection, together with the relevance of Matrix Metalloproteases (MMPs) -1, -2, -7, -9 and -10 in lung tumorigenesis, prompted us to assess the diagnostic usefulness of these MMPs and the Tissue Inhibitor of Metalloproteinase (TIMP) -1 in NSCLC patients.
\end{abstract}

Methods: Markers were evaluated in an initial study cohort (19 NSCLC cases and 19 healthy controls). Those that better performed were analyzed in a larger sample including patients with benign lung diseases. Serum MMPs and TIMP-1 were determined by multiplexed immunoassays. Logistic regression was employed for multivariate analysis of biomarker combinations.

Results: MMPs and TIMP-1 were elevated in the serum of NSCLC patients compared to healthy controls. MMP-1, -7 and -9 performed at best and were further evaluated in the sample including benign pathologies, corroborating the superiority of MMP-9 in NSCLC discrimination, also at early-stage NSCLC. The optimal diagnostic value was obtained with the model including MMP-9, gender, age and smoking history, that demonstrated an AUC of 0.787, $85.54 \%$ sensitivity and $64.89 \%$ specificity.

Conclusion: Our results suggest that MMP-9 is a potential biomarker for NSCLC diagnosis and its combined measurement with other biomarkers could improve NSCLC detection.

Keywords: Matrix metalloproteases, Non-small cell lung cancer, Diagnosis, Serum biomarkers

\section{Background}

Non-small cell lung cancer (NSCLC) accounts for 75$80 \%$ of the newly diagnosed lung cancers and includes the main histological subtypes adenocarcinoma (ADC), squamous cell carcinoma (SCC) and large cell carcinoma (LCC) [1]. NSCLC 5-year survival rates around 13\% [2] make essential an improvement of prognosis, which can be achieved with the detection of cancer at early stages. Consequently, there is an imperative need of noninvasive tests, preferably blood-based biomarkers that could be used as tools for the early detection of lung cancer $[3,4]$.

Matrix metalloproteases (MMPs) constitute a large family of structurally related, zinc- and calcium-dependent

\footnotetext{
*Correspondence: Idechiara@uvigo.es

'Department of Biochemistry, Genetics and Immunology, Universidade de

Vigo.Vigo, As Lagoas-Marcosende s/n, 36310 Vigo, Spain

Full list of author information is available at the end of the article
}

enzymes capable of degrading almost all of the extracellular matrix (ECM) proteins. These endopeptidases have been widely associated with the development of various diseases, including cancer [5]. The over-expression of MMPs induced by both tumor cells and surrounding stroma is not limited to matrix degradation, favoring invasion and metastasis (reviewed in $[6,7])$. Through the activation of nonmatrix substrates such as growth factors, cytokines and other membrane proteins, MMPs are also involved in initial stages of tumor development mediating signaling pathways related to cell migration, differentiation, proliferation, apoptosis, angiogenesis and inflammatory reactions [8].

Numerous studies have demonstrated that MMPs are specifically implicated in lung-tumorigenesis driven processes, contributing to the formation of a complex microenvironment promoting malignant transformation in lung tissue (reviewed in [9]). MMP-1 has proved to be a tumor growth promoting and pro-angiogenic factor in 
the lungs of MMP-1-deficient mice [10]. MMP-2 holds a role in lung cancer angiogenesis mediated by vascular endothelial growth factor expression [11] and also in the invasive behavior of tumor cells, as for MMP-7 [12]. A common role in metastasis has been attributed to MMP-9, triggered by MMP-9 induction in the premetastatic lung by other distant primary tumors [13]. Regarding MMP-10, over-expression in cancer-initiating or cancer stem cells is key on their maintenance and tumorigenic potential, allowing for lung tumor-initiating activity and metastatic spread [14].

The functions of MMPs are controlled by the four Tissue Inhibitors of Metalloproteinases, TIMP-1 - 4 [15]. The local balance between MMPs and TIMPs is critical to avoid the conditions of uncontrolled ECM turnover, inflammation, and deregulated cell growth and migration, which would result in disease [16].

The involvement of the aforementioned MMPs and their inhibitors in lung carcinogenesis makes this group of molecules attractive as potential markers for NSCLC. Our objective was to determine the serum levels of the selected MMPs (MMP-1, -2, -7, -9 and -10) and TIMP-1 in NSCLC patients and healthy and benign controls to investigate their capability for NSCLC discrimination.

\section{Methods}

\section{Study population}

Patients with respiratory symptoms were prospectively enrolled at the Department of Pneumology of Hospital Álvaro Cunqueiro EOXI Vigo (Spain) between June 2007 and June 2011. Diagnosis and classification of lung cancer patients were based on the clinical guidelines of the American College of Chest Physicians [17]. Patients with non-NSCLC histology, relapse or progression of a previously diagnosed cancer, or administration of radiotherapy/chemotherapy were excluded from the study.

Initial evaluation of MMPs and TIMP-1 was conducted in a reduced cohort of 19 NSCLC cases and 19 healthy controls, whose demographics are presented in Additional file 1. Markers exhibiting greater discriminative capability were determined in a larger sample set including 193 individuals: 83 NSCLC cases (48.2\% ADC, $31.3 \%$ SCC and $16.9 \%$ LCC) and 110 controls (75 individuals with benign lung pathology and 35 healthy patients). Characteristics of NSCLC patients and controls are summarized in Table 1. Smoking status was defined 'Yes' for current smokers and ex-smokers, and 'No' for never-smokers.

Blood samples from all patients and controls were collected at their first visit to the Department of Pneumology and serum was separated and stored at $-20{ }^{\circ} \mathrm{C}$ until analysis. The study was conducted in compliance with the clinical-ethical practices of the Spanish Government and the Helsinki Declaration, and Galician Ethical
Table 1 Patient demographics

\begin{tabular}{|c|c|c|}
\hline & Cases $(n=83)$ & Controls $(n=110)$ \\
\hline \multicolumn{3}{|l|}{ Gender $^{a}$} \\
\hline Male & 68 (81.9\%) & $62(56.4 \%)$ \\
\hline Female & 15 (18.1\%) & $48(43.6 \%)$ \\
\hline \multicolumn{3}{|l|}{ Age $^{\mathrm{b}}$} \\
\hline Median & 69 & 61 \\
\hline Range & $42-88$ & $24-88$ \\
\hline \multicolumn{3}{|l|}{ Smoking status ${ }^{c}$} \\
\hline Yes & 74 (89.2\%) & $62(66 \%)$ \\
\hline No & $9(10.8 \%)$ & $32(33 \%)$ \\
\hline \multicolumn{3}{|l|}{ Diagnosis } \\
\hline Healthy & & 35 \\
\hline Benign Pathology & & 75 \\
\hline RI & & $53(70.7 \%)$ \\
\hline ILD & & 17 (22.6\%) \\
\hline Nodule & & $4(5.3 \%)$ \\
\hline ICC & & $1(1.3 \%)$ \\
\hline \multicolumn{3}{|l|}{ NSCLC Histology } \\
\hline$A D C$ & 40 (48.2\%) & \\
\hline SCC & $26(31.3 \%)$ & \\
\hline LCC & $14(16.9 \%)$ & \\
\hline BAC & $2(2.4 \%)$ & \\
\hline ND & $1(1.2 \%)$ & \\
\hline \multicolumn{3}{|l|}{ NSCLC Stage } \\
\hline I & $18(21.7 \%)$ & \\
\hline$\|$ & $6(7.2 \%)$ & \\
\hline III & $22(26.5 \%)$ & \\
\hline IV & 37 (44.6\%) & \\
\hline
\end{tabular}

RI Respiratory Infection, ILD Interstitial Lung Disease, ICC Congestive Heart Failure, NSCLC Non-Small Cell Lung Cancer, ADC Adenocarcinoma, SCC Squamous Cell Carcinoma, LCC Large Cell Carcinoma, BAC Bronchioloalveolar Carcinoma, ND Not Differentiated Carcinoma

${ }^{a}$ Gender distribution between cancer and controls statistically significant: $P<0.001$ (Fisher test)

${ }^{b}$ Statistically significant differences in age between cancer and controls: $P=0.001$ (Mann-Whitney U test)

'Smoking status distribution between cancer and controls statistically different: $P<0.001$ (Fisher test)

Committee for Clinical Research approved the protocol. Written informed consent from each patient was obtained.

\section{Measurement of serum MMPs and TIMP-1 concentration} Serum MMPs and TIMP-1 determination was carried out by means of multiplexed immunoassays with Luminex xMAP technology (EMD Millipore, Missouri, USA). Measurements of MMP-1, MMP-2, MMP-7, MMP-9 and MMP-10 were conducted with the commercially available Human MMP Panel 2 Magnetic Bead kit (HMMP2MAG, EMD Millipore) according to the manufacturer protocol, 
while TIMP-1 was part of the Human TIMP Panel 1 Magnetic Bead kit (HTMP1MAG, EMD Millipore).

Fluorescence readings were collected on a Luminex platform (Luminex $200^{\mathrm{rm}}$ ), and calculation of results was performed using the BioPlex Manager ${ }^{\mathrm{Tm}}$ software (Bio-Rad, Hercules, CA), with protein concentrations calculated using a 5-parametric curve fitting. Both standard and serum samples were assayed in duplicate to reduce variation.

\section{Statistical methods}

Descriptive statistics were obtained for continuous (median and range) and categorical variables (frequencies). Differences in serum biomarker concentrations were assessed using the non-parametric Mann-Whitney $U$ test. The Fisher exact test was applied to determine the association between qualitative variables. To analyze the diagnostic accuracy of the biomarkers for NSCLC diagnosis, Receiver Operating Characteristic (ROC) curves were calculated, providing the area under the ROC curve (AUC). $P$-values $\leq 0.05$ were considered statistically significant.

Univariate logistic regression was performed to evaluate the individual capability of biomarkers and demographic variables of predicting lung malignancy. Logistic regression models, using log10-tranformed marker concentrations to reduce skewness, were employed for multivariate analysis of biomarker combinations. Models were constructed with all possible combinations of selected markers to determine the optimal marker set. Age and gender were included in the regression models to adjust for confounding. Predicted probabilities of malignancy for each individual, generated by the logistic function, were used to calculate the diagnostic performance of the assayed marker combinations, providing the AUC and the sensitivity and specificity based on the Youden index. Statistical analyses were carried out with the statistical software SPSS 15.0 (SPSS Inc., Chicago, IL); sensitivity and specificity were calculated using the MedCalc software.

\section{Results}

Analysis of serum MMPs and TIMP-1 in NSCLC patients and healthy controls: Initial study set

Members of the MMP family MMP-1, $-2,-7,-9$ and -10 and the MMP-9 inhibitor TIMP-1 were first assayed in the initial study set to select the molecules with best performance.

All MMPs and TIMP-1 exhibited elevated levels in cancer patients (Table 2). However, concentrations were

Table 2 Serum Levels of MMPs and TIMP-1 in Non-Small Cell Lung Cancer and Healthy Controls in the Initial Study Set

\begin{tabular}{|c|c|c|c|c|}
\hline Marker $^{a}$ & Median & Range & $p^{b}$ & AUC (95\% Cl) \\
\hline \multicolumn{5}{|c|}{ MMP-1 (pg/mL) } \\
\hline Healthy & 4504.00 & $1186.61-16,751.12$ & \multirow[t]{2}{*}{0.015} & \multirow[t]{2}{*}{$0.729(0.560-0.897)$} \\
\hline NSCLC & 8739.00 & $2517.57-20,715.00$ & & \\
\hline \multicolumn{5}{|c|}{ MMP-2 (pg/mL) } \\
\hline Healthy & $84,644.00$ & $76,006.00-117,129.00$ & \multirow[t]{2}{*}{0.584} & \multirow[t]{2}{*}{$0.446(0.251-0.641)$} \\
\hline NSCLC & $85,643.00$ & $60,249.00-144,375.00$ & & \\
\hline \multicolumn{5}{|c|}{ MMP-7 (pg/mL) } \\
\hline Healthy & $16,964.00$ & $11,409.14-27,067.99$ & \multirow[t]{2}{*}{0.120} & \multirow[t]{2}{*}{$0.658(0.473-0.843)$} \\
\hline NSCLC & $20,742.11$ & $7256.00-50,354.00$ & & \\
\hline \multicolumn{5}{|c|}{ MMP-9 (ng/mL) } \\
\hline Healthy & 136.14 & $63.13-492.91$ & \multirow[t]{2}{*}{$<0.001$} & \multirow[t]{2}{*}{$0.852(0.725-0.979)$} \\
\hline NSCLC & 302.92 & $131.16-824.08$ & & \\
\hline \multicolumn{5}{|c|}{ MMP-10 (pg/mL) } \\
\hline Healthy & 350.00 & 132.00-993.00 & \multirow[t]{2}{*}{0.533} & \multirow[t]{2}{*}{$0.564(0.355-0.774)$} \\
\hline NSCLC & 400.00 & 209.00-730.00 & & \\
\hline \multicolumn{5}{|c|}{ TIMP-1 (ng/mL) } \\
\hline Healthy & 130.45 & $92.40-238.90$ & \multirow[t]{2}{*}{0.191} & \multirow[t]{2}{*}{$0.625(0.443-0.806)$} \\
\hline NSCLC & 151.65 & $110.05-241.00$ & & \\
\hline \multicolumn{5}{|c|}{ MMP-9/TIMP1 } \\
\hline Healthy & 1.09 & $0.51-5-05$ & \multirow[t]{2}{*}{0.002} & \multirow[t]{2}{*}{$0.781(0.628-0.934)$} \\
\hline NSCLC & 2.42 & $0.73-4.80$ & & \\
\hline
\end{tabular}


only significantly higher for MMP-1 $(P=0.015)$, MMP-9 $(P<0.001)$ and MMP-9/TIMP1 ratio $(P=0.002)$, despite MMP-7 showed notably higher levels in NSCLC. ROC curves proved the superiority of MMP-9 for discriminating NSCLC cases with an AUC of 0.852, followed by MMP-9/TIMP1 (AUC 0.781), MMP-1 (AUC 0.729) and MMP-7 with a modest discriminatory capacity (AUC 0.658). Correction of MMP-9 levels by its inhibitor levels failed to improve the diagnostic performance of the matrix metalloproteinase member, therefore TIMP1 was not further evaluated.

\section{Analysis of serum MMP-1, -7 and -9 in NSCLC and control patients}

After identifying MMP-1, -7 and -9 as the MMPs with major contribution to NSCLC detection, they were further evaluated in a larger set consisting of 83 NSCLC cases, 35 healthy controls and 75 patients with benign lung pathology.

The relationship between demographic variables and marker concentrations were established, as presented in Table 3. Analysis showed significant differences in serum MMP-9 regarding gender, with levels being higher in males $(P=0.010)$. In relation to age, MMP-7 levels were found to be significantly higher in older individuals $(P<$ 0.001). Smoking history did not significantly influence levels of any of the markers, although notable higher MMP-9 concentrations occurred in smokers in relation to subjects who have never smoked.

Statistically significant higher serum levels were confirmed for MMP-7 $(P=0.014)$ and MMP-9 $(P<0.001)$ when comparing lung cancer with non-cancer patients (both healthy and benign). Increased concentration of MMP-1 in benign controls, compared to healthy individuals, accounts for the lack of significance. In addition, when NSCLC and benign controls were compared, only MMP-9 resulted significantly altered $(P<0.001)$. These findings, including the median values for markers in the different diagnostic groups, are provided in Table 4.

In terms of AUC, MMP-9 was the marker that better discriminated NSCLC from all control individuals (AUC 0.739), while MMP-1 and MMP-7 demonstrated poor diagnostic capability (AUC of 0.538 and 0.604, respectively).

\section{Analysis of serum MMP-1, MMP-7 and MMP-9 regarding NSCLC stage}

Marker levels were also evaluated according to cancer stage (Table 4). A trend towards increased marker levels was observed with cancer progression, particularly for MMP-1 and MMP-9. Consequently, all three MMPs exhibited significant differences between late-stage NSCLC and all controls. MMP-9 was the only molecule that could discriminate NSCLC at early stages from control individuals $(P=0.002)$, suggesting its usefulness in early NSCLC diagnosis.

\section{Multi-MMP panel for NSCLC classification}

Prior to evaluate the usefulness of the combination of MMPs for NSCLC discrimination, univariate logistic regression models of MMPs and demographic variables was accomplished to assess their predictive value for NSCLC (Additional file 2). Univariate regression demonstrated a significant association between being male, older aged, current or past smoking history, and high levels of MMP members MMP-7 and MMP-9. As expected, the strongest relation with lung malignancy was observed for MMP-9, with an unadjusted OR of 8.86 (95\% CI 3.17-24.76). When the predictive variables were included in multivariate regression models, MMP-9 remained the unique MMP significant in NSCLC prediction, as well as older age and having a positive smoking history.

Since MMP-9 resulted the marker that better discriminated cancer from controls, this molecule was combined with MMP-1 and/or -7 with the intention of increasing its diagnostic performance. Logistic regression was applied to the 177 patients with data on MMP-1, MMP-7 and MMP-9, and information on gender, age and smoking variables, to construct multimarker classification algorithms including gender, age and smoking history as confounders. Discriminatory capability by means of AUC was estimated for MMP-9 and each combination,

Table 3 Relationship Between Serum Levels of MMP-1, -7 and -9 and Demographic Parameters

\begin{tabular}{|c|c|c|c|c|c|c|c|c|c|}
\hline \multirow[t]{2}{*}{ Marker } & \multicolumn{3}{|l|}{ Gender $^{a}$} & \multicolumn{3}{|l|}{$\mathrm{Age}^{\mathrm{a}}$} & \multicolumn{3}{|l|}{ Smoking History ${ }^{\text {a, c }}$} \\
\hline & Male $(n=130)$ & Female $\left(n=63^{\circ}\right)$ & $p^{b}$ & $\begin{array}{l}\leq 64 \text { years } \\
(n=97)\end{array}$ & $\begin{array}{l}>64 \text { years } \\
(n=96)\end{array}$ & $p^{b}$ & Yes $(n=136)$ & No $(n=41)$ & $p^{b}$ \\
\hline \multirow[t]{2}{*}{ MMP-1 (pg/mL) } & 6061.80 & 7572.03 & 0.799 & 6146.60 & 6384.94 & 0.682 & 5872.18 & 7683.08 & 0.700 \\
\hline & $1207.70-41,668.33$ & $935.32-24,851.96$ & & $1081.81-32,677.57$ & $935.32-41,668.33$ & & $1271.24-41,668.33$ & $935.32-24,851.96$ & \\
\hline \multirow[t]{2}{*}{ MMP-7 (pg/mL) } & $24,238.56$ & $23,035.24$ & 0.777 & $20,810.85$ & $27,700.03$ & $<0.001$ & $24,411.99$ & $22,974.55$ & 0.349 \\
\hline & $5026.14-79,977.27$ & $5383.18-72,191.16$ & & $5026.14-72,191.16$ & $5383.18-79,977.27$ & & $5026.14-79,977.27$ & $5383.18-59,232.82$ & \\
\hline \multirow[t]{2}{*}{ MMP-9 (ng/mL) } & 273.18 & 201.75 & 0.010 & 228.04 & 260.42 & 0.477 & 265.73 & 201.75 & 0.060 \\
\hline & $21.06-3611.59$ & $11.07-3300.50$ & & $11.07-3611.59$ & $52.79-1883.70$ & & $21.06-3611.59$ & $11.07-3300.50$ & \\
\hline
\end{tabular}

Median and range values provided

${ }^{\mathrm{b}}$ Mann-Whitney $\mathrm{U}$ test for the comparison between gender, age and smoking groups 
Table 4 Serum Levels of MMP-1, -7 and -9 in Non-Small Cell Lung Cancer and Controls

\begin{tabular}{|c|c|c|c|c|}
\hline Marker $^{a}$ & Median & Range & $p^{b}$ & AUC (95\% Cl) \\
\hline \multicolumn{5}{|l|}{ MMP-1 (pg/mL) } \\
\hline Control & 6209.72 & $935.32-23,960.37$ & 0.364 & \multirow[t]{6}{*}{$0.538(0.457-0.620)$} \\
\hline Healthy & 4730.11 & $1081.81-23,635.33$ & 0.045 & \\
\hline Benign & 7483.33 & $935.32-23,960.37$ & 0.974 & \\
\hline NSCLC & 6653.85 & $1450.34-41,668.33$ & & \\
\hline NSCLC I + II & 6046.96 & $1784.41-30,180.33$ & 0.772 & \\
\hline NSCLC III + IV & 6972.73 & $1450.34-41,668.33$ & 0.324 & \\
\hline \multicolumn{5}{|l|}{ MMP-7 (pg/mL) } \\
\hline Control & $22,130.10$ & $5026.14-79,977.27$ & 0.014 & \multirow[t]{6}{*}{$0.604(0.523-0.685)$} \\
\hline Healthy & $21,010.07$ & $8893.61-48,743.79$ & 0.004 & \\
\hline Benign & $22,739.58$ & $5026.14-79,977.27$ & 0.109 & \\
\hline NSCLC & $26,485.45$ & $5383.18-79,809.13$ & & \\
\hline $\mathrm{NSCLCI}+\|$ & $26,165.87$ & $13,727.30-54,573.51$ & 0.465 & \\
\hline NSCLC III + IV & $26,680.26$ & $5383.18-79,809.13$ & 0.007 & \\
\hline \multicolumn{5}{|l|}{ MMP-9 (ng/mL) } \\
\hline Control & 194.84 & $11.07-3611.59$ & $<0.001$ & \multirow[t]{6}{*}{0.739 (0.670-0.809) } \\
\hline Healthy & 177.86 & $52.79-800.30$ & $<0.001$ & \\
\hline Benign & 200.28 & $11.07-3611.59$ & $<0.001$ & \\
\hline NSCLC & 348.04 & 21.06-1941.08 & & \\
\hline NSCLC I + II & 346.03 & 116.92-1941.08 & 0.002 & \\
\hline NSCLC III + IV & 364.62 & 21.06-1914.00 & $<0.001$ & \\
\hline
\end{tabular}

NSCLC Non-Small Cell Lung Cancer

a Sample size: Control $N=110$ (Healthy $N=35$, Benign $N=75), \operatorname{NSCLC~} N=83(\mathrm{NSCLC} I+\| N=24, \mathrm{NSCLC}$ III + IV N=59)

${ }^{\mathrm{b}}$ Mann-Whitney $\mathrm{U}$ test for the comparison between the cancer and control groups, for subtypes of controls versus NSCLC, and NSCLC stages versus all controls

as well as the diagnostic parameters based on the Youden index (Table 5).

The AUC for MMP-9 adjusted by gender, age and smoking history (0.787) practically did not increase when combined with either MMP-1, MMP-7 or both MMP-1 and MMP-7 (AUC of 0.787,0.786 and 0.788, respectively), indicating that these MMPs do not complement MMP-9 to separate cancer patients from controls.
In relation to the diagnostic parameters, MMP-9 combination with demographic characteristics rendered notable sensitivity and specificity (85.54 and 64.89\%, respectively), identical to those obtained when MMP-1 is included in the classification model. On the other hand, the inclusion of MMP-7 or MMP-1 and MMP-7 diminished sensitivity (81.93\%) and specificity (64.52\%) in both cases.

Table 5 Comparison of Diagnostic Performance of MMP-9 and its Combination with MMP-1 and/or MMP-7 for Non-Small Cell Lung Cancer Detection

\begin{tabular}{|c|c|c|c|c|}
\hline Model $^{a}$ & AUC & $\begin{array}{l}\text { Sensitivity \% } \\
(95 \% \mathrm{Cl})^{\mathrm{b}}\end{array}$ & $\begin{array}{l}\text { Specificity \% } \\
(95 \% \text { Cl) b }\end{array}$ & Youden Index \\
\hline$M M P-9+G n+A g+S m k$ & $0.787(0.720-0.845)$ & $\begin{array}{l}85.54 \\
(76.1-92.3)\end{array}$ & $\begin{array}{l}64.89 \\
(54.4-74.5)\end{array}$ & $>0.419$ \\
\hline$M M P-9+M M P-1+G n+A g+S m k$ & $0.787(0.720-0.845)$ & $\begin{array}{l}85.54 \\
(76.1-92.3)\end{array}$ & $\begin{array}{l}64.89 \\
(54.4-74.5)\end{array}$ & $>0.421$ \\
\hline$M M P-9+M M P-7+G n+A g+S m k$ & $0.786(0.718-0.844)$ & $\begin{array}{l}81.93 \\
(72-89.5)\end{array}$ & $\begin{array}{l}64.52 \\
(53.9-74.2)\end{array}$ & $>0.413$ \\
\hline MMP-9 + MMP-1 + MMP-7 + Gn $+\mathrm{Ag}+\mathrm{Smk}$ & $0.788(0.720-0.845)$ & $\begin{array}{l}81.93 \\
(72-89.5)\end{array}$ & $\begin{array}{l}64.52 \\
(53.9-74.2)\end{array}$ & $>0.415$ \\
\hline
\end{tabular}

Gn Gender, Ag Age, Smk Smoking history

a Logistic regression models were elaborated including the 177 patients with data on MMP-1, MMP-7 and MMP-9, and information on gender, age and smoking variables

${ }^{\mathrm{b}}$ Sensitivity and specificity calculated based on Youden Index 


\section{Discussion}

Initially MMPs were considered only relevant to invasion and metastasis due to their relation with the cleavage of structural components of the ECM. However, through their action on other non-ECM substrates, including growth factors and receptors, cytokines, chemokines and cell-adhesion molecules, they regulate important cancer processes such as cell growth, apoptosis, tumor angiogenesis and evasion of immune surveillance $[5,18]$.

On the basis of their lung-tumorigenesis promoting functions [10-14], the MMP members MMP-1, MMP-2, MMP-7, MMP-9 and MMP-10 may result promising biomarkers for lung cancer. Furthermore, these MMPs are commonly reported as over-expressed in lung tumor tissues as compared to corresponding normal tissues in NSCLC patients [19-22], however, little is known about their diagnostic value. The goal of this study was to evaluate serum MMP-1, -2, -7, -9, -10 and TIMP-1 in discriminating NSCLC from non-cancerous controls.

Two of the five MMPs first assayed in the initial sample population were discarded based on poor AUC values (MMP-2: 0.446; MMP-10: 0.564), showing no value for NSCLC discrimination. The work of Kanoh et al. [23] compared serum MMP-2 levels in NSCLC patients and healthy controls, finding no significant alteration in MMP-2 concentration. In relation to serum MMP-10, its potential as a tumor marker for NSCLC diagnosis has not been earlier addressed, to our knowledge. Available literature reports higher expression in lung tumor tissues [22], although reduced MMP-10 mRNA levels have been reported too [24].

MMP-1, MMP-7 and MMP-9 in the initial study set described relatively elevated serum levels in NSCLC patients versus controls, and that prompted us to determine their diagnostic value in a larger population comprising benign pathologies of the lung that must also be discerned from NSCLC patients. Overall, MMP-1 levels in NSCLC were higher than that of the control group, although discrimination was poor as evidenced by the AUC value of 0.538 . MMP-1 studies on its diagnostic usefulness for NSCLC are scarce. The study of Li et al. [25] in plasma of lung cancer patients confirmed higher MMP-1 levels in NSCLC regarding healthy individuals, but diagnostic capability seemed limited too. Clearly, MMP-1 concentration is enhanced in the benign group, abrogating the discrimination from NSCLC, in agreement with the observation of Rosas et al. [26], who propose MMP-1 as a blood marker for Idiophatic Pulmonary Fibrosis (IPF). Regarding tumor stage, higher levels were found in advanced stages versus controls, although this increase did not reach statistical difference. Li et al. [25] also observed considerable higher levels for stages III and IV, finding an association of both elevated plasma levels and tissue up-regulation with poor survival rates. These results suggest that MMP-1 could be implicated in the late stages of the tumor process.

Determination of MMP-7 levels in NSCLC, benign and healthy controls displayed a statistical significant increase in cancer patients, though a moderate discriminatory capacity was found for MMP-7 (AUC: 0.604). As for MMP-1, studies on MMP-7 point to a role of this MMP as marker of IPF rather than NSCLC diagnosis $[26,27]$. Ulivi et al. [27] analyzed the diagnostic potential of MMP-7 in discriminating between NSCLC and other lung diseases (chronic obstructive pulmonary disease, asthma,...), healthy donors and non-specific interstitial pneumonias. In spite of significantly higher levels in NSCLC than in lung diseases and donors, similar levels to that of interstitial pneumonias derived in an AUC of 0.64.

MMP-9 is one of the most studied MMPs in lung cancer, with several reports addressing its potential as diagnostic marker. In our study, significantly elevated serum MMP-9 was observed in patients with malignancy regarding both healthy controls and patients with benign pathologies, offering the best diagnostic capacity for NSCLC with an AUC value of 0.739. These findings are in line with other studies reporting significantly higher MMP-9 levels in serum of NSCLC patients compared with non-malignant lung diseases or healthy controls [28-30]. Especially relevant diagnostic terms are presented in the study by Zhang et al. [28], who reported an AUC of 0.882 in differentiating NSCLC from healthy and non-malignant lung patients, and by Fiorelli et al. [29], who proposed the combined use of MMP-9 with PET to diagnose malignancy in indeterminate pulmonary lung lesions. This study described for PET-negative patients a sensitivity of $94 \%$ and specificity of $100 \%$ in classifying these lesions.

Regarding tumor stage we found a not significant increase in MMP-9 levels with advanced stages. These results are consistent with the described trend of highest levels in metastatic disease [29, 30]. The association between serum MMP-9 and gender was also previously reported [28], with males presenting higher levels.

The balance between MMPs production and their regulators is crucial to avoid deregulated ECM turnover or cell growth, which would result in disease $[16,18]$. In this work we also aimed to consider the effect of TIMP1 on MMP-9 levels by defining the ratio MMP-9/TIMP1 in the initial study set. We found that TIMP-1 levels were moderately elevated in NSCLC patient's sera, as previously observed by Jumper et al. [30], although these authors found a significant increase. Nevertheless, the MMP-9/TIMP-1 ratio did not improve the metalloprotease discrimination of NSCLC, with a loss in AUC from 
0.852 to 0.781 , therefore it was not further evaluated. The relationship between MMP-9 and its inhibitor remains undetermined, as both no correlation [30] and a significant correlation [31] have been reported in serum of lung cancer patients, and could be in part attributable to other non-MMP inhibiting activities of TIMP-1.

Among all the MMPs analyzed in our study, including the MMP-9/TIMP-1 ratio, MMP-9 displayed the best diagnostic accuracy for NSCLC. Disappointingly, this discrimination seems not good enough for clinical application. Numerous reports in the literature have evaluated the utility of combined tumor-related markers for lung cancer detection [32-34] and, consequently, we tested whether MMP-1, MMP-7 and MMP-9 could provide complementary discrimination and improve the performance of MMP-9. Logistic regression was used to develop panels composed of 2 or 3 markers, in conjunction with the variables gender and age, to distinguish between NSCLC cases and controls. Two-marker panels based on MMP-9 with either MMP-1 or MMP-7 offered identical AUCbased discrimination, but did not improve that of MMP-9 adjusted by confounders.

Several publications have recently examined the additional value of serum MMPs to protein markers on NSCLC detection models, as Farlow et al. [35] and Bigbee et al. [36]. The first included MMP-2 as part of a multi-analyte test for prediction of NSCLC diagnosis, reporting significantly higher concentrations in the control group compared to NSCLC, with an AUC value of 0.705 . Inclusion of rheumatology inflammatory conditions and resected nodules as non-cancerous controls could account for the discrepancy with our results. That study also evaluated MMP-9 and TIMP-1 among the candidate markers, though these were not selected as statistically relevant by the algorithm employed. Another multiplexed-marker panel for complementing CT in discriminating NSCLC from cancer-free controls was proposed by Bigbee et al. [36]. Among the 70 cancerassociated proteins evaluated, MMP members MMP-1, -7 and -9 were considered, although they were not included in the final diagnostic rule. On the contrary, Izbicka et al. [37] in plasma from NSCLC and healthy controls proposed a 5 -marker panel whose diagnostic performance reached $95 \%$ sensitivity and $79 \%$ specificity, resulting MMP-9 one of the relevant proteins in the discriminative model.

\section{Conclusions}

Our study on the relevance of MMPs in NSCLC diagnosis indicates that MMP-2 and MMP-10 exhibit a poor discrimination of NSCLC versus healthy controls, while MMP-1 and -7 differentiate NSCLC from healthy controls, but not from benign diseases. On the other hand, a notable discriminatory performance was reached for MMP-9 when determined jointly with demographic variables. Based on this evidence, MMP-9 would be a suitable candidate to be included in other models or assays to improve the accuracy in NSCLC diagnosis.

\section{Additional files}

Additional file 1: Patient Demographics and Classification of Non-Small Cell Lung Cancer in Initial Study Set. (DOC 43 kb)

Additional file 2: Univariate and multivariate logistic regression analysis of MMP-1, MMP-7, MMP-9, gender, age and smoking history regarding NSCLC. (DOCX $13 \mathrm{~kb})$

\section{Abbreviations}

ADC: Adenocarcinoma; AUC: Area under the ROC curve; ECM: Extracellular matrix; IPF: Idiopathic pulmonary fibrosis; LCC: Large cell carcinoma; MMPs: Matrix metalloproteases; NSCLC: Non-small cell lung cancer; ROC: Receiver operating characteristic; SCC: Squamous cell carcinoma; TIMP: Tissue inhibitors of metalloproteinases

\section{Acknowledgements}

Not applicable.

\section{Funding}

This study was supported by Instituto de Salud Carlos III (PS09-00405), and Xunta de Galicia (INBIOMED 2012-273 and GRC2014/019, FEDER support included). S. Blanco-Prieto was supported by Spanish Ministry of Science and Innovation (fellowship FPU). The samples used in this work belong to the Biobank from EOXI Vigo (RETIC-FISISCIIIRD09/0076/00011).

\section{Availability of data and materials}

The datasets available from the corresponding author on reasonable request.

\section{Authors' contributions}

Conception and design: MP, LD, FJRB, AFV; Acquisition of data: SBP, LBC, MBR; Analysis and Interpretation: SBP, LD, LVI, LBC, MP; Drafting the manuscript for important intellectual content: LD, SBP, MP; Critical revisions: All authors; Approval of the final version: All authors.

Ethics approval and consent to participate

The study was conducted in compliance with the clinical-ethical practices of the Spanish Government and the Helsinki Declaration, and Galician Ethical Committee for Clinical Research approved the protocol. Written informed consent from each patient was obtained.

\section{Consent for publication}

Not required.

\section{Competing interests}

The authors declare that they have no competing interest.

\section{Publisher's Note}

Springer Nature remains neutral with regard to jurisdictional claims in published maps and institutional affiliations.

\section{Author details}

'Department of Biochemistry, Genetics and Immunology, Universidade de Vigo.Vigo, As Lagoas-Marcosende s/n, 36310 Vigo, Spain. ${ }^{2}$ Department of Pneumology of Hospital Álvaro Cunqueiro EOXI Vigo, Carretera Clara Campoamor, 341, 36212 Vigo, Spain. 
Received: 12 January 2017 Accepted: 24 November 2017 Published online: 05 December 2017

\section{References}

1. Rivera MP, Mehta AC, Wahidi MM. Establishing the diagnosis of lung cancer: diagnosis and management of lung cancer, 3rd ed: American college of chest physicians evidence-based clinical practice guidelines. Chest. 2013; doi:10.1378/chest.12-2353.

2. Francisci S, Minicozzi P, Pierannunzio D, Ardanaz E, Eberle A, Grimsrud TK, et al. Survival patterns in lung and pleural cancer in Europe 1999-2007: results from the EUROCARE-5 study. Eur J Cancer. 2015; doi:10.1016/j.ejca.2015.07.033.

3. Hensing TA, Salgia R. Molecular biomarkers for future screening of lung cancer. J Surg Oncol. 2013; 10.1002/jso.23382.

4. I H CJY. Lung cancer biomarkers. Adv Clin Chem. 2015; doi:10.1016/bs.acc. 2015.07.003

5. Egeblad M, Werb Z. New functions for the matrix metalloproteinases in cancer progression. Nat Rev Cancer. 2002; doi:10.1038/nrc745.

6. Roy R, Yang J, Moses MA. Matrix metalloproteinases as novel biomarkers and potential therapeutic targets in human cancer. J Clin Oncol. 2009; doi:10.1200/JCO.2009.23.5556

7. Martin MD, Matrisian LM. The other side of MMPs: protective roles in tumor progression. Cancer Metastasis Rev. 2007; doi:10.1007/s10555-0079089-4.

8. Hadler-Olsen E, Winberg JO, Uhlin-Hansen L. Matrix metalloproteinases in cancer: their value as diagnostic and prognostic markers and therapeutic targets. Tumor Biol. 2013; doi:10.1007/s13277-013-0842-8.

9. Gueders MM, Foidart JM, Noel A, Cataldo DD. Matrix metalloproteinases (MMPs) and tissue inhibitors of MMPs in the respiratory tract: potential implications in asthma and other lung diseases. Eur J Pharmacol. 2006; doi:10.1016/j.ejphar.2005.12.082

10. Foley CJ, Fanjul-Fernández M, Bohm A, Nguyen N, Agarwal A, Austin K, et al. Matrix metalloprotease 1a deficiency suppresses tumor growth and angiogenesis. Oncogene. 2014; doi:10.1038/onc.2013.157.

11. Chetty C, Lakka SS, Bhoopathi P, Rao JS. MMP-2 alters VEGF expression via aVB3 integrin-mediated PI3K AKT signaling in A549 lung cancer cells. Int J Cancer. 2010; doi:10.1002/ijc.25134.

12. Kim EJ, Lee SY, Woo MK, Choi SI, Kim TR, Kim MJ, et al. Fibulin-3 promoter methylation alters the invasive behavior of non-small cell lung cancer cell lines via MMP-7 and MMP-2 regulation. Int J Oncol. 2012; doi:10.3892/ijo.2011.1191.

13. Hiratsuka S, Nakamura K, Iwai S, Murakami M, Itoh T, Kijima H, et al. MMP9 induction by vascular endothelial growth factor receptor-1 is involved in lungspecific metastasis. Cancer Cell. 2002; doi:10.1016/S1535-6108(02)00153-8.

14. Justilien V, Regala RP, Tseng IC, Walsh MP, Batra J, Radisky ES, et al. Matrix metalloproteinase-10 is required for lung cancer stem cell maintenance, tumor initiation and metastatic potential. PLoS One. 2012; doi:10.1371/ journal.pone.0035040.

15. Deryugina El, Quigley JP. Matrix metalloproteinases and tumor metastasis. Cancer Metastasis Rev. 2006; doi:10.1007/s10555-006-7886-9.

16. Löffek S, Schilling O, Franzke CW. Series "matrix metalloproteinases in lung health and disease": biological role of matrix metalloproteinases: a critical balance. Eur Respir J. 2011; doi:10.1183/09031936.00146510.

17. Alberts WM; American College of Chest Physicians. Diagnosis and management of lung cancer executive summary. ACCP evidence-based clinical practice guidelines (2nd edition). Chest. 2007; doi: 10.1378/chest.07-1860

18. Kessenbrock K, Plaks V, Werb Z. Matrix Metalloproteinases: regulators of the tumor microenvironment. Cell. 2010; doi:10.1016/j.cell.2010.03.015.

19. Shah SA, Spinale FG, Ikonomidis JS, Stroud RE, Chang El, Reed CE. Differential matrix metalloproteinase levels in adenocarcinoma and squamous cell carcinoma of the lung. J Thorac Cardiovasc Surg. 2010; doi:10.1016/j.jtcvs.2009.12.016.

20. Gouyer V, Conti M, Devos P, Zerimech F, Copin MC, Créme E, et al. Tissue inhibitor of metalloproteinase 1 is an independent predictor of prognosis in patients with nonsmall cell lung carcinoma who undergo resection with curative intent. Cancer. 2005; doi:10.1002/cncr.20965.

21. Leinonen T, Pirinen $R$, Böhm J, Johansson R, Ropponen $K$, Kosma VM. Expression of matrix metalloproteinases 7 and 9 in non-small cell lung cancer: relation to clinicopathological factors, B-catenin and prognosis. Lung Cancer. 2006; doi:10.1016/j.lungcan.2005.11.002.

22. doi:Gill JH, Kirwan IG, Seargent JM, Martin SW, Tijani S, Anikin VA, et al. MMP-10 is overexpressed, proteolytically active, and a potential target for therapeutic intervention in human lung carcinomas. Neoplasia. 2004; doi:10.1593/neo.04283.

23. Kanoh Y, Abe T, Masuda N, Akahoshi T. Progression of non-small cell lung cancer: diagnostic and prognostic utility of matrix metalloproteinase-2, C-reactive protein and serum amyloid a. Oncol Rep. 2013; doi:10.3892/or.2012.2123.

24. Zhang X, Zhu S, Luo G, Zheng L, Wei J, Zhu J, et al. Expression of MMP-10 in lung cancer. Anticancer Res. 2007;27:2791-5.

25. Li M, Xiao T, Zhang Y, Feng L, Lin D, Liu Y, et al. Prognostic significance of matrix metalloproteinase-1 levels in peripheral plasma and tumour tissues of lung cancer patients. Lung Cancer. 2010; doi:10.1016/j.lungcan.2009.12.007.

26. Rosas IO, Richards TJ, Konishi K, Zhang Y, Gibson K, Lokshin AE, et al. MMP1 and MMP7 as potential peripheral blood biomarkers in idiopathic pulmonary fibrosis. PLoS Med. 2008; doi:10.1371/journal.pmed.0050093.

27. Ulivi P, Casoni GL, Foschi G, Scarpi E, Tomassetti S, Romagnoli M, et al. MMP-7 and fCDNA serum levels in early NSCLC and idiopathic interstitial pneumonia: preliminary study. Int J Mol Sci. 2013; doi:10.3390/ ijms141224097.

28. Zhang Y, Wu JZ, Zhang JY, Xue J, Ma R, Cao HX, et al. Detection of circulating vascular endothelial growth factor and matrix metalloproteinase9 in non-small cell lung cancer using Luminex multiplex technology. Oncol Lett. 2014; doi:10.3892/ol.2013.1718.

29. Fiorelli A, Rizzo A, Messina G, Izzo A, Vicidomini G, Pannone G, et al. Correlation between matrix metalloproteinase 9 and 18F-2-fluoro-2deoxyglucose-positron emission tomography as diagnostic markers of lung cancer. Eur J Cardiothorac Surg. 2012; doi:10.1093/ejcts/ezr117.

30. Jumper C, Cobos E, Lox C. Determination of the serum matrix metalloproteinase-9 (MMP-9) and tissue inhibitor of matrix metalloproteinase-1 (TIMP-1) in patients with either advanced small-cell lung cancer or non-small-cell lung cancer prior to treatment. Respir Med. 2004;98:173-7.

31. Ylisirniö S, Höyhtyä M, Mäkitaro R, Pääakkö P, Risteli J, Kinnula VL, et al. Elevated serum levels of type I collagen degradation marker ICTP and tissue inhibitor of metalloproteinase (TIMP) 1 are associated with poor prognosis in lung cancer. Clin Cancer Res. 2001;7:1633-7.

32. Doseeva V, Colpitts T, Gao G, Woodcock J, Knezevic V. Performance of a multiplexed dual analyte immunoassay for the early detection of non-small cell lung cancer. J Transl Med. 2015; doi:10.1186/s12967-015-0419-y.

33. Blanco-Prieto $S$, Vázquez-Iglesias $L$, Rodríguez-Girondo M, Barcia-Castro L, Fernández-Villar A, Botana-Rial MI, et al. Serum calprotectin, CD26 and EGF to establish a panel for the diagnosis of lung cancer. PLoS One. 2015; doi:10.1371/journal.pone.0127318.

34. Jett JR, Peek LJ, Fredericks L, Jewell W, Pingleton WW, Robertson JF. Audit of the autoantibody test, EarlyCDT ${ }^{\circledR}$-lung, in 1600 patients: an evaluation of its performance in routine clinical practice. Lung Cancer. 2014; doi:10.1016/j. lungcan.2013.10.008

35. Farlow EC, Vercillo MS, Coon JS, Basu S, Kim AW, Faber LP, et al. A multianalyte serum test for the detection of non-small cell lung cancer. $\mathrm{Br}$ J Cancer. 2010; doi:10.1038/sj.bjc.6605865.

36. Bigbee WL, Gopalakrishnan V, Weissfeld JL, Wilson DO, Dacic S, Lokshin AE, et al. A multiplexed serum biomarker immunoassay panel discriminates clinical lung cancer patients from high-risk individuals found to be cancerfree by CT screening. J Thorac Oncol. 2012; doi:10.1097/JTO. 0b013e31824ab6b0.

37. Izbicka E, Streeper RT, Michalek JE, Louden CL, Diaz A 3rd, Campos DR. Plasma biomarkers distinguish non-small cell lung cancer from asthma and differ in men and women. Cancer Genomics Proteomics. 2012;9:27-35. 